\title{
A Continuous-Time Search Model with Job Switch and Jumps
}

\author{
Masahiko Egami*
}

Mingxin $\mathrm{Xu}^{\dagger}$

\begin{abstract}
We study a new search problem in continuous time. In the traditional approach, the basic formulation is to maximize the expected (discounted) return obtained by taking a job, net of search cost incurred until the job is taken. Implicitly assumed in the traditional modeling is that the agent has no job at all during the search period or her decision on a new job is independent of the job situation she is currently engaged in. In contrast, we incorporate the fact that the agent has a job currently and starts searching a new job. Hence we can handle more realistic situation of the search problem. We provide optimal decision rules as to both quitting the current job and taking a new job as well as explicit solutions and proofs of optimality. Further, we extend to a situation where the agent's current job satisfaction may be affected by sudden downward jumps (e.g. de-motivating events), where we also find an explicit solution; it is rather a rare case that one finds explicit solutions in control problems using a jump diffusion.
\end{abstract}

Key words: search problem, Poisson arrivals, optimal stopping, jump diffusion Mathematics Subject Classification (2000) : Primary: 60G40 Secondary:60G35

\section{Introduction}

In this paper a continuous-time search problem is discussed in which offers of random size are received randomly over time. This work is positioned in the vein of research papers by Lippman and McCall [14], Zuckerman [21, 22, 23], and Stadje [20]. In these papers, the case of a Poisson-model offer is analyzed and subsequently the results are generalized to the case where the arrival times form a renewal process. In a typical setting, the problem is written as

$$
g_{T}=\mathbb{E}\left[e^{-\alpha T} Y(T)-\int_{0}^{T} c e^{-\alpha t} d t\right]
$$

to find the stopping time $T$ that maximizes the right hand side with $Y(t)$ being the stochastic process interpreted as the highest offer received up to time $t$. Stadje [20] extended to the case where the rate of arrivals and search

\footnotetext{
*Graduate School of Economics, Kyoto University, Kyoto, 606-8501, Japan. Email:egami@econ.kyoto-u.ac.jp.

${ }^{\dagger}$ Department of Mathematics and Statistics, University of North Carolina at Charlotte, NC. 28223 U.S.A. Email:mxu2@uncc.edu. The work of Mingxin Xu is supported by National Science Foundation under the grant SES-0518869 and John H. Biggs Faculty Fellowship.
} 
cost are both time-dependent. Further extensions of the studies of this problem are Boshuizen and Gouweleeuw $[4,5,6]$. Boshuizen and Gouweleeuw [4] study optimal stopping problems for semi-Markov processes. In the context of job search problems, this setting allows interarrival times of job offers to be transition-dependent. In other words, the time until the next offer arrives depends on the highest offer that the agent has obtained up to now. In [5], the semi-Markov model is treated as a special case of multivariate point processes. Using the results from the preceding papers, the search problems are fully discussed in [6] where they provide, under general interarrival time distributions (in a finite and an infinite time horizon), the recursive (dynamic programming) formulae for the value function and the characterization of optimal strategies. Hence the main results obtained by Zuckerman in $[22,23]$ are reproduced as special cases. These studies are, among other things, in the direction of generalizing the structure of interarrival times and of deriving appropriate dynamic programming equations for the solution. The basic formulation is to maximize the expected (discounted) return obtained by taking a job, net of search cost incurred until the job is taken. Implicitly assumed is that the agent has no job at all during the search period or her decision on a new job is independent of the current job situation.

We extend the search problems to some other directions in this paper. The agent has a job and considers a new job (by quitting the current one.) Our model discussed in this paper allows the agent to start searching for a new job while she is being employed. Hence the agent shall determine when to quit the current job and when to take a new job offer. We model the state of a current job by a one-dimensional diffusion and formulate an optimal stopping problem where the decision variables are both the time of quitting the current job and the time of taking a new offer. We assume that the interarrival times are exponentially distributed and attempt to obtain explicit form solutions. After we describe the model, we solve a restricted case where search can start only after the agent quits her current job (Section 2.1). Second, we relax this constraint so that the agent can start job search while she is being employed (Section 2.2). Third, an extension is attempted to the case where the state of a current job is described by a jump diffusion (Section 3). Stochastic jumps represent sudden deterioration of job environments. In each problem, we provide an optimal search rule, its proof and explicit solutions. The first two cases are solved in a quite general setting. In the jump diffusion setting, we apply a method with which we directly identify the value function and, thereby, are able to significantly facilitate the process of finding the optimal solutions.

This paper adds some new material to the literature of search problem. Other directions of the search problem in the recent literature include Nakai [16] that studies in a partially observable Markov chain setting, and Collins and McNamara [9] where a "secretary problem" is analyzed under competition in an infinite population of candidates and an infinite population of posts of diverse value.

\section{Model}

Let $(\Omega, \mathcal{F}, \mathbb{P})$ be a complete probability space with a standard Brownian motion $B=\left\{B_{t}: t \geq 0\right\}$ and a Poisson Process $N=\left\{N_{t}: t \geq 0\right\}$ independent of $B$. Let us consider the diffusion process $X=\left\{X_{t}: t \geq 0\right\}$ in the following form:

$$
d X_{t}=\mu\left(X_{t}\right) d t+\sigma\left(X_{t}\right) d B_{t}, \quad X_{0}=x,
$$

with state space $\mathcal{I} \subseteq \mathbb{R}$, which is assumed to be an interval with endpoints $-\infty \leq a<b \leq \infty$. The drift and diffusion coefficients $\mu(\cdot): \mathcal{I} \rightarrow \mathbb{R}$ and $\sigma: \mathcal{I} \rightarrow(0, \infty)$ are some Borel functions and we assume (2.1) has weak solution with unique probability law, which is guaranteed, for example, if

$$
\forall x \in \operatorname{int}(\mathcal{I}), \exists \epsilon>0 \quad \text { such that } \int_{(x-\epsilon, x+\epsilon)} \frac{1+|\mu(y)|}{\sigma^{2}(y)} d y<\infty .
$$


See Section 5.5 of Karatzas and Shreve [12]. We also assume that $X$ is regular, that is, $X$ reaches, from any $x \in(a, b)$, another point $y \in \mathcal{I}$ with positive probability. Let $\alpha \geq 0$ be a real constant and $f(\cdot)$ be a continuous function that satisfies

$$
\mathbb{E}^{x}\left[\int_{0}^{\infty} e^{-\alpha s}\left|f\left(X_{s}\right)\right| d s\right]<\infty
$$

Let $Y=\left\{Y_{t_{1}}, Y_{t_{2}} \ldots\right\}$ be the sequence of independently and identically distributed positive random variables observable at time $t_{1}, t_{2}, \ldots$ from the common distribution $G$ with finite mean $E(Y)<\infty$ and independent of Brownian motion $B$. The arrivals of these random variables $Y$ 's follow an independent Poisson process $N=$ $\{N(t): t \geq 0\}$ with rate $\lambda>0$. We assume that there is no offer available at time 0 . That is, $N(0)=0$. We denote by $\mathbb{F}=\left\{\mathcal{F}_{t}\right\}_{t \geq 0}$ the filtration generated by $X$ and $Y$. We write $Y_{t}$ as the value that the agent observes when it appears at time $t$ and the agent cannot hold the offers. Since $Y$ is available only at the times of Poisson arrivals, it is quite natural to consider the following optimal stopping problems:

The first problem (P1): Consider the following functional

$$
v(x):=\sup _{\zeta, \tau \in \mathcal{S}_{1}} \mathbb{E}^{x}\left[\int_{0}^{\zeta} e^{-\alpha s} f\left(X_{s}\right) d s+e^{-\alpha \zeta} k\left(X_{\zeta}\right)-\int_{\zeta}^{\tau} c e^{-\alpha s} d s+e^{-\alpha \tau} Y_{\tau}\right],
$$

where $\mathcal{S}_{1}$ is the set of admissible $\mathbb{F}$-stopping times. In our first problem, the set $\mathcal{S}_{1}$ is of the form:

$$
\mathcal{S}_{1}:=\{\zeta<\tau: \zeta, \tau \in \mathcal{S}\}
$$

where we denote by $\mathcal{S}$ all the $\mathbb{F}$-stopping times. $k: \mathbb{R} \rightarrow \mathbb{R}$ is the terminal payoff function incurred at time $\zeta$. The constant $c>0$ is the rate of search cost.

We can view the process $X$ as the state of the current job. It is possible to view $f(\cdot)$ as a utility function. The agent obtains monetary value and/or satisfaction level $f\left(X_{t}\right)$ that arises from her current job at time $t$. Since the agent will be offered other job opportunities in the market, she always has an option to quit the job at time $\zeta$ and switch to another one, at time $\tau$, whose wage is expressed by $Y_{\tau}$. However, in this setting, she starts searching only after she quits the incumbent. At time $\zeta$, she receives or pays the amount represented by $k(\cdot)$ based on the state of the current job. This amount, if negative, can be interpreted as a hurdle of switching the current job. In other words, if $x \rightarrow k(x)$ is decreasing in $x$ (for example, $k(x)=b x, b<0$ ), then the agent has small incentive to change jobs when the state variable $X$ is large because she has to experience large negative utility.

It should be emphasized that this setting can be easily applied to property sales (house, manufacturing plant, or entire business). In the alternative setting, the stochastic process $X$ represents the state of the current house, $f(\cdot)$ translates the state into economic value, $k(\cdot)$ denotes expenditures incurred at the sale and finally $Y$ 's are the stream of offers given to the house.

The second problem (P2): We relax the restriction of the order of $\zeta<\tau$ in the second problem.

$$
v(x)=\sup _{\zeta, \tau \in \mathcal{S}_{2}} \mathbb{E}^{x}\left[\int_{0}^{\zeta} e^{-\alpha s} f\left(X_{s}\right) d s+e^{-\alpha \zeta} k\left(X_{\zeta}\right)-\int_{0}^{\tau} c e^{-\alpha s} d s+e^{-\alpha \tau} Y_{\tau}\right]
$$

where

$$
\mathcal{S}_{2}:=\{\zeta \leq \tau: \zeta, \tau \in \mathcal{S}\}
$$

In other words, the agent starts searching for a new job while she is being employed. Note that the lower limit of the integral for search cost is zero. If $\tau$ occurs (at $\zeta$ ), then she quits the current job and takes the new one simultaneously. If $\zeta$ occurs (before $\tau$ ), then she quits the current job and keeps searching for a new job. The latter case reduces to the first problem (P1). 


\subsection{Solution to the first problem}

Our solution to the first problem (P1) will be explained by a series of lemmas along with steps that simplify (2.3). First we set $J^{\zeta, \tau}(x):=\mathbb{E}^{x}\left[\int_{0}^{\zeta} e^{-\alpha s} f\left(X_{s}\right) d s+e^{-\alpha \zeta} k\left(X_{\zeta}\right)-\int_{\zeta}^{\tau} c e^{-\alpha s} d s+e^{-\alpha \tau} Y_{\tau}\right]$ for our performance measure. We have

$$
\begin{aligned}
J^{\zeta, \tau}(x) & =\mathbb{E}^{x}\left[\int_{0}^{\zeta} e^{-\alpha s} f\left(X_{s}\right) d s+e^{-\alpha \zeta} k\left(X_{\zeta}\right)-\int_{\zeta}^{\tau} c e^{-\alpha s} d s+e^{-\alpha \tau} Y_{\tau}\right] \\
& =\mathbb{E}^{x}\left[\left(\int_{0}^{\infty}-\int_{\zeta}^{\infty}\right) e^{-\alpha s} f\left(X_{s}\right) d s-\int_{\zeta}^{\tau} c e^{-\alpha s} d s+e^{-\alpha \zeta} k\left(X_{\zeta}\right)+e^{-\alpha \tau} Y_{\tau}\right] \\
& =\mathbb{E}^{x}\left[g(x)-e^{-\alpha \zeta} g\left(X_{\zeta}\right)+e^{-\alpha \zeta} k\left(X_{\zeta}\right)+e^{-\alpha \zeta} \mathbb{E}^{X_{\zeta}}\left[\int_{0}^{\tau} e^{-\alpha s}(-c) d s+e^{-\alpha \tau} Y_{\tau}\right]\right] .
\end{aligned}
$$

In the last line, we denote

$$
g(x):=\mathbb{E}^{x}\left[\int_{0}^{\infty} e^{-\alpha s} f\left(X_{s}\right) d s\right]
$$

and use, for the first two terms containing $X$, the strong Markov property of $X$ by noting that $X$ and $Y$ are independent and by conditioning on the value of $X_{\zeta}$. We also use the fact that $\mathbb{P}(\tau>\zeta)=1$ for the last two terms of (2.7). Indeed, the search starts at time $\zeta$ in this problem. It can be further simplified to

$$
\begin{aligned}
J^{\zeta, \tau}(x) & =\mathbb{E}^{x}\left[g(x)-e^{-\alpha \zeta} g\left(X_{\zeta}\right)+e^{-\alpha \zeta} k\left(X_{\zeta}\right)+e^{-\alpha \zeta} \mathbb{E}^{X_{\zeta}}\left[-\frac{c}{\alpha}+e^{-\alpha \tau} \frac{c}{\alpha}+e^{-\alpha \tau} Y_{\tau}\right]\right] \\
& =\mathbb{E}^{x}\left[g(x)+e^{-\alpha \zeta}\left\{k\left(X_{\zeta}\right)-g\left(X_{\zeta}\right)-\frac{c}{\alpha}+\mathbb{E}\left[e^{-\alpha \tau}\left(\frac{c}{\alpha}+Y_{\tau}\right)\right]\right\}\right]
\end{aligned}
$$

by the independence of $X$ and $Y$ again with the memoryless property of Poisson arrival time for the last term of (2.9) at the last step. Hence we can split the problem into two stages: first maximizing over $\tau$ to obtain a constant from the last term and then secondly maximizing over $\zeta$, namely

$$
v(x)=\sup _{\zeta \in \mathcal{S}_{1}} \mathbb{E}^{x}\left[g(x)+e^{-\alpha \zeta}\left\{k\left(X_{\zeta}\right)-g\left(X_{\zeta}\right)-\frac{c}{\alpha}+\sup _{\tau \in \mathcal{S}_{1}} \mathbb{E}\left[e^{-\alpha \tau}\left(\frac{c}{\alpha}+Y_{\tau}\right)\right]\right\}\right] .
$$

Let us consider the first stage optimization, the inner maximization. Since $c / \alpha$ is a constant, we only have to consider the following problem: Let us consider a situation that the agent observes a stream of $i$. $i$. $d$. random variables $Y$ with Poisson interarrival. Our task is to set an optimal stopping rule. Call this auxiliary problem $\left(P^{\prime} 1\right)$. Suppose that the current value the agent observes is $y$. Then, the dynamic programming equation is simple in this case;

$$
V(y):=\max (y, V)
$$

where $V$ is the maximum expected discounted value if $y$ is rejected. That is, $V(y)$ is the maximum expected return when there is current offer $y$ available. See standard textbooks, for example, Ross [18]. Also see Ross [19] that contains many examples including search models.

Now let $V_{0}(y):=y$ and for $n>0$,

$$
V_{n}(y):=\max \left(y, \mathbb{E}\left[e^{-\alpha \tau^{(1)}} \int_{0}^{\infty} V_{n-1}(z) G(d z)\right]\right)
$$

where $\tau^{(1)}$ is the first jump time of the Poisson process and $G(\cdot)$ is the distribution of the offers. Hence $V_{n}(y)$ is the maximum reward if we observe $y$ now and are allowed a maximum of $n$ offers before stopping. Then we have $V_{n}(y) \leq V_{n+1}(y) \leq V(y)$, by construction, for all $y \geq 0$. 
Let us define

$$
B:=\left\{i \in \mathbb{R}_{+}: i \geq \mathbb{E}\left[e^{-\alpha \tau^{(1)}} Y_{\tau^{(1)}}\right]\right\}
$$

$B$ is the set of states for which stopping is at least as good as continuing for one more period (round) and then stopping. The policy that stops the first time the process enters a state in $B$ is referred to as one-stage lookahead policy. Let us write $P_{i j}$ for the transitional probability of process $Y$ for a general argument, while $P_{i j}$ is independent of $i$ in our problem.

Lemma 2.1. For auxiliary problem $\left(P^{\prime} 1\right)$, if the process is stable in the sense that $\lim _{n \rightarrow \infty} V_{n}(y)=V(y)$ for all $y \geq 0$ and if $P_{i j}=0$ for $i \in B, j \notin B$, then optimal policy stops at $i$ if and only if $i \in B$.

Proof. This is in essence Theorem 2.2 (page 54) in [18]. Here we show it for completeness. It should be shown that $V_{n}(i)=i$ for all $i \in B$ and all $n$. It follows for $n=0$ and let us suppose it for $n-1$ for induction. Then for $i \in B$,

$$
\begin{aligned}
V_{n}(i) & =\max \left\{i, \mathbb{E}\left[e^{-\alpha \tau^{(1)}} \int_{0}^{\infty} V_{n-1}(z) G(d z)\right]\right\} \\
& =\max \left\{i, \mathbb{E}\left[e^{-\alpha \tau^{(1)}} \int_{z \in B} V_{n-1}(z) G(d z)\right]\right\} \\
& =\max \left\{i, \mathbb{E}\left[e^{-\alpha \tau^{(1)}} \int_{z \in B} z G(d z)\right]\right\}=i
\end{aligned}
$$

where the second equality is due to the structure of $B$ and the third due to the induction hypothesis. Hence $V_{n}(i)=i$ for all $i \in B$ and all $n$. By letting $n \rightarrow \infty$ and using the stability assumption, we obtain $V(i)=i$ for $i \in B$.

On the other hand, for $i \notin B$, the policy that continues for one more stage and stops has an expected reward, $\mathbb{E}\left[e^{-\alpha \tau^{(1)}} \int_{0}^{\infty} z G(d z)\right]$ which is strictly greater than $i$ since $i \notin B$. Summarizing, we have $V(i)=i$ for $i \in B$ and $V(i)>i$ for $i \notin B$.

Lemma 2.2. If $Y$ is a positive random variable and $\mathbb{E}(Y)<\infty$, then $\lim _{n \rightarrow \infty} V_{n}(y)=V(y)$ for all $y \in \mathbb{R}_{+}$.

Proof. Since $V_{n}(y) \leq V(y)$ for all $n \geq 0$, we only need to prove the opposite direction. Let $S^{*}$ be the set of all the strategies. Consider two strategies that are identical up to $\tau^{(n)}$. One strategy keeps observing $Y_{n+1}, Y_{n+2}, \ldots$ We call the present value (at zero) of this strategy $V^{*}(y)$. The other strategy stops with $V_{n}(y)$. Call the set of these strategies $S_{n}$. We have $S_{n} \subset S^{*}$. For notational convenience, let us define no-discount versions of $V_{n}(\cdot)$. Namely, $\bar{V}_{0}(y)=y, \bar{V}_{n}(y)=\max \left(y, \int_{0}^{\infty} V_{n-1}(z) G(d z)\right)$ and we discount from time $\tau^{(n)}$ to time zero to get $V_{n}(y)$. Now let us denote by $\bar{V}$ a value of the first strategy discounted back up to time $\tau^{(n)}$. Without loss of generality, we can state $\bar{V} \geq \bar{V}_{n}(y)$ for all $n$. Indeed, if it were not the case, we would have $\sup _{\nu \in S^{*}} \bar{V} \leq \bar{V}_{n}(y)$, leading to the desired inequality $V_{n}(y) \geq V(y)$ and the proof would be complete. Hence we have

$$
\left|V^{*}(y)-V_{n}(y)\right| \leq \mathbb{E}\left[e^{-\alpha \tau^{(n)}}\left|\bar{V}-\bar{V}_{n}(y)\right|\right] \leq \mathbb{E}\left[e^{-\alpha \tau^{(n)}} \bar{V}\right] .
$$

Since $\mathbb{E}(Y)<\infty$ and $Y$ is positive, $\lim _{s \rightarrow \infty} \mathbb{P}(Y>s)=0$, implying that we have $\lim _{x \rightarrow \infty} \mathbb{P}(\bar{V}>x)=0$. By passing $n$ to the limit, the right hand side becomes zero by the dominated convergence theorem. Hence we have $V(y)=\sup _{\nu \in S^{*}} V^{*}(y)=\sup _{\nu \in \cup_{n} S_{n}} V_{n}(y)$. This shows that $\lim _{n \rightarrow \infty} V_{n}(y) \geq V(y)$.

Let us call the set that satisfies the assumption of this lemma, "closed" set. We now come back to our original problem (P1). In applying this lemma to our problem at hand, we need to modify the "closedness" of $B$. Since any offer can be declined, the assumption of the lemma does not hold. However, we have the following result. 
Lemma 2.3. An optimal policy is to accept the first offer that is at least $u^{*}$ where

$$
u^{*}:=\min \left\{i: \frac{\alpha i+c}{\lambda} \geq \mathbb{E}\left[(Y-i)^{+}\right]\right\} .
$$

The optimal threshold $u^{*}$ exists and is unique if and only if $\lambda \mathbb{E}(Y) \geq c$.

Proof. Let us allow the agent to recall any past offer, that is, the current offer is always the running maximum of the offers up to present. Going back to (2.3), we redefine our closed (in $i$ ) set $B$ and incorporating the recallability of past offers, we have

$$
\begin{aligned}
B & =\left\{i: i \geq \mathbb{E}\left[e^{-\alpha \tau^{(1)}} Y_{\tau^{(1)}}-\int_{0}^{\tau^{(1)}} c e^{-\alpha s} d s\right]\right\} \\
& =\left\{i: i \geq \frac{\lambda}{\alpha+\lambda}\left(\int_{0}^{i} i G(d y)+\int_{i}^{\infty} y G(d y)\right)-\left(\frac{c}{\alpha}-\frac{\lambda}{(\alpha+\lambda)} \frac{c}{\alpha}\right)\right\} \\
& =\left\{i: i \geq \frac{\lambda}{\alpha+\lambda}\left(i-i \int_{i}^{\infty} G(d y)+\int_{i}^{\infty} y G(d y)\right)-\frac{c}{\alpha}+\frac{\lambda}{(\alpha+\lambda)} \frac{c}{\alpha}\right\} \\
& =\left\{i: \alpha i \geq \lambda \int_{i}^{\infty}(y-i) G(d y)-c\right\}=\left\{i: \frac{\alpha i+c}{\lambda} \geq \mathbb{E}\left[(Y-i)^{+}\right]\right\},
\end{aligned}
$$

which is (2.13). The left hand side is a linear function in $i$ with positive slope $\alpha / \lambda$ and positive intercept $c / \lambda$. The right hand side is a decreasing function of $i$ and it is $\mathbb{E}(Y)>0$ at $i=0$. Hence equation (2.13) has unique solution if and only if $\mathbb{E}(Y) \geq c / \lambda$.

For $i>u^{*}, i \in B$. This fact implies that once $i$ (that carries the running maximum of the offers) enters set $B$, it does not leave set $B$. It is because the running maximum cannot decrease in time. Hence $B$ is "closed". Although we cannot recall past offers in our problem, this policy (2.13) is still feasible in the original problem (no recall). Now, it was shown that the first time $Y$ enters in $B$ gives us an optimal policy in a larger set of strategies (namely including the recalling). Hence this policy cannot be beaten by any other strategies in the smaller set (allowed in the original problem). It follows that (2.13) is an optimal policy.

Economically speaking, this condition says that, if we would consider $\sum_{i}^{N(t)} Y_{i}$ as a compound Poisson just for interpretation purposes, the search is meaningful if the expected value of the compound Poisson per unit time $\lambda E(Y)$ is greater than the search cost per unit time $c$. Moreover, if we look at the left hand side $\frac{\alpha i+c}{\lambda}$, we conclude that a large discount rate $\alpha$, a large rate of search cost $c$ and a small frequency of job offers $\lambda$ will lead to a small optimal acceptance level $u^{*}$, which fits with our intuition.

Remark 2.1. The papers we mentioned in Section 1 assume the recallability and prove the optimality of the one-stage look-ahead strategy. This argument of "closedness" of set $B$ reaches the same conclusion under the assumption of no recall.

Now we shall show a convenient result for computing (2.10):

Lemma 2.4. Suppose that the distribution of offers has the density with support in $(0, \infty)$ and $\lambda \mathbb{E}(Y) \geq c$, then

$$
\sup _{\tau \in \mathcal{S}} \mathbb{E}\left[e^{-\alpha \tau}\left(\frac{c}{\alpha}+Y_{\tau}\right)\right]=\frac{c}{\alpha}+u^{*}
$$

where $u^{*}$ is the unique solution to

$$
\frac{\alpha u^{*}+c}{\lambda}=E\left[\left(Y-u^{*}\right)^{+}\right]
$$


Proof. Let us denote $\overline{G(} \cdot)=1-G(\cdot)$ where $G(\cdot)$ is the distribution of offers. We use the thinning argument of the Poisson process. Since the agent follows the strategy described in Lemma 2.3, for any $u>0, \tau$ is the first arrival time of the offer greater than or equal to $u$. The agent is interested in a sequence of random variables $Y 1_{\{Y \geq u\}}$ and this thinned Poisson process has rate $\lambda \bar{G}(u)$. Due to the independence of $Y$ and $N(t)$ in the original setting, we have the independence of $Y 1_{\{Y \geq u\}}$ and the thinned Poisson process with rate $\lambda \bar{G}(u)$, given the offer is greater than or equal to $u$.

$$
\begin{aligned}
\mathbb{E}\left[e^{-\alpha \tau}\left(\frac{c}{\alpha}+Y_{\tau}\right)\right] & =\mathbb{E}\left[e^{-\alpha \tau}\left(\frac{c}{\alpha}+Y\right) \mid Y \geq u\right] \\
& =\frac{c}{\alpha} \int_{0}^{\infty} \lambda \bar{G}(u) e^{-\alpha t} e^{-\lambda \bar{G}(u) t} d t+\frac{\int_{0}^{\infty} \int_{u}^{\infty} y G(d y) e^{-\alpha t} \lambda \bar{G}(u) e^{-\lambda \bar{G}(u) t} d t}{\bar{G}(u)} \\
& =\frac{c \lambda \bar{G}(u)}{\alpha(\alpha+\lambda \bar{G}(u))}+\frac{\lambda}{\alpha+\lambda \bar{G}(u)} \int_{u}^{\infty} y G(d y),
\end{aligned}
$$

which is a function of $u$ only. By differentiating with respect to $u$, we obtain the first order condition for optimality

$$
\left(-\frac{c}{\alpha} g(u)-u g(u)\right)(\alpha+\lambda \bar{G}(u))+\lambda\left(\frac{c}{\alpha} \bar{G}(u)+\int_{u}^{\infty} y g(y) d y\right) g(u)=0 .
$$

This can further be simplified to

$$
\int_{u}^{\infty}(y-u) g(y) d y=\frac{\alpha u+c}{\lambda} .
$$

The integral on the left hand side is just $\mathbb{E}\left[(Y-u)^{+}\right]$and the above equation is the same as (2.15). Denote by $u^{*}$ the solution of (2.17) and write $\mathbb{E}\left[\left(Y-u^{*}\right)^{+}\right]=\mathbb{E}\left[Y 1_{\left\{Y \geq u^{*}\right\}}\right]-u^{*} \bar{G}\left(u^{*}\right)=\frac{\alpha u^{*}+c}{\lambda}$. This is equivalent to

$$
u^{*}+\frac{c}{\alpha+\lambda \bar{G}\left(u^{*}\right)}=\frac{\lambda \mathbb{E}\left[Y 1_{\left\{Y \geq u^{*}\right\}}\right]}{\alpha+\lambda \bar{G}\left(u^{*}\right)} .
$$

By plugging (2.18) in (2.16) and noting that $u^{*}$ is the optimal threshold level, we have

$$
\sup _{\tau \in \mathcal{S}_{1}} \mathbb{E}\left[e^{-\alpha \tau}\left(\frac{c}{\alpha}+Y_{\tau}\right)\right]=\frac{c \lambda \bar{G}(u)}{\alpha(\alpha+\lambda \bar{G}(u))}+u^{*}+\frac{c}{\alpha+\lambda \bar{G}\left(u^{*}\right)}=u^{*}+\frac{c}{\alpha} .
$$

Now that the first stage optimization is done and the last term of (2.10) becomes constant, it reduces to an optimal stopping problem to find $\zeta^{*}$. By using Lemma 2.3, (2.10) is now in the following simple form:

$$
\bar{v}(x):=v(x)-g(x)=\sup _{\zeta \in \mathcal{S}_{1}} \mathbb{E}^{x}\left[e^{-\alpha \zeta}\left(k\left(X_{\zeta}\right)-g\left(X_{\zeta}\right)+u^{*}\right)\right] .
$$

Notice that $c / \alpha$ term is canceled. This equation does not have any $c$ explicitly, but we have to keep in mind that the solution to (2.19) does depend on $c$ because $u^{*}$ depends on $c$ through (2.15).

For the solution of (2.19), let us use the characterization of the value function of optimal stopping problems in one-dimension by Dynkin [11] and/or Dayanik and Karatzas [10]. Let us denote by $\psi(\cdot)$ and $\varphi(\cdot)$ the increasing and decreasing functions of the solution of $(\mathcal{A}-\alpha) w(\cdot)=0$ with $\mathcal{A}$ being the infinitesimal generator of $X$. Let us define

$$
F(x):=\psi(x) / \varphi(x) .
$$

We use the transformation for a Borel function $h(\cdot): \mathbb{R} \rightarrow \mathbb{R}$,

$$
H(\cdot):=h\left(F^{-1}(\cdot)\right) / \varphi\left(F^{-1}(\cdot)\right) .
$$


For our problem, let us define

$$
h(x):=k(x)-g(x)+u^{*}
$$

and

$$
l_{c}:=\limsup _{x \rightarrow c} \frac{(h(x))^{+}}{\varphi(x)},
$$

where $(h(x))^{+}=\max (h(x), 0)$. Theoretically, we can compute $\bar{v}(x)$ for any $f, k, G(d y)$ and the underlying diffusion $X$. But it is useful if we provide sufficient conditions that the optimal continuation region (that is, no job-quitting region) is a connected interval in the real line:

Proposition 2.1. Suppose that the state space $(c, d) \subseteq \mathbb{R}$ of $X$ has natural boundaries at $c$ and $d$ with $l_{c}<\infty$. If $H_{0}(y):=h\left(F^{-1}(y)\right) / \varphi\left(F^{-1}(y)\right)$ has sole local maximum in the interior of $(F(c), F(d))$ with $\lim _{y \rightarrow c} H_{0}^{\prime \prime}(y)<0$ and $\lim _{y \rightarrow d} H_{0}^{\prime \prime}(y)>0$, then the value function, that is the solution to (P1) in (2.3), is of the form

$$
v(x)= \begin{cases}k(x)+u^{*}, & x \leq x^{*}, \\ v_{0}(x):=p \cdot \varphi(x)+g(x), & x>x^{*},\end{cases}
$$

where $p$ and $x^{*}$ are uniquely determined.

Proof. Define $y=F(x)$ and denote $W(y)$ as the smallest concave majorant of

$$
H(y):= \begin{cases}H_{0}(y), & y>0 \\ l_{c}, & y=0 .\end{cases}
$$

By Proposition 5.12 in [10], $\bar{v}(x)=\varphi(x) W(F(x))$ and $W(0)=l_{c}(W(\cdot)$ is continuous at $y=0)$ and the optimal stopping rule is given by $\tau^{*}=\inf \left\{t \geq 0: X_{t} \in \Gamma\right\}$ where $\Gamma:=\{x \in(c, d): v(x)=h(x)\}$. Under our assumptions, $H(y)$ has a global maximum at which the function is concave (call this point $y^{*}$ ) and becomes convex on $(\bar{y}, \infty)$ where $\bar{y}>y^{*}$. Then the smallest concave majorant $W(y)$ is $H(y)$ itself on $\left(0, y^{*}\right)$ and the horizontal line passing point $\left(y^{*}, H\left(y^{*}\right)\right)$. Call $p:=H\left(y^{*}\right)$. Transforming back to the original space, $\bar{v}(x)=h(x)$ on $\left(c, F^{-1}\left(y^{*}\right)\right)$ and $\bar{v}(x)=p \varphi(x)$. Adding back $g(x)$ to obtain $v(x)$, which is the solution.

Remark 2.2. Note that as we describe in our problem statement at the beginning of this section, in practice $f(\cdot)$ is increasing and $k(\cdot)$ is decreasing in the state variable. Hence if $g(\cdot)$ is also increasing, $h(\cdot)=k(\cdot)-g(\cdot)+u^{*}$ is decreasing in state. The example below is a typical one of this case.

Example 2.1. The solution can be best illustrated by a simple example. Our example is $X_{t}=B_{t}$ with $B_{0}=x$ and $f(x)=a x$ with $a>0, k(x)=b x$ with $b<0$ and $c=0$. Here we assume that $G(d y)=m e^{-m y} d y$. Hence $u^{*}$ satisfies

$$
\frac{e^{-m u}}{m}=\frac{\alpha u}{\lambda}
$$

from (2.17). Then our $g(x)=\frac{a}{\alpha} x$ and problem (2.3) becomes

$$
\begin{aligned}
\bar{v}(x) & =\sup _{\zeta \in \mathcal{S}_{1}} \mathbb{E}^{x}\left[e^{-\alpha \zeta}\left\{\left(b-\frac{a}{\alpha}\right) x+\frac{\lambda}{\alpha+\lambda \bar{G}\left(u^{*}\right)} \int_{u^{*}}^{\infty} y G(d y)\right\}\right] \\
& =\sup _{\zeta \in \mathcal{S}_{1}} \mathbb{E}^{x}\left[e^{-\alpha \zeta}\left\{\left(b-\frac{a}{\alpha}\right) x+u^{*}\right\}\right] .
\end{aligned}
$$




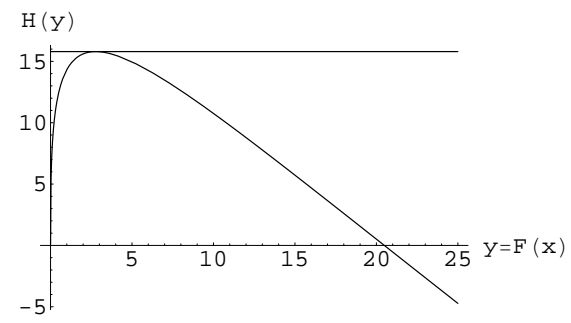

(a)

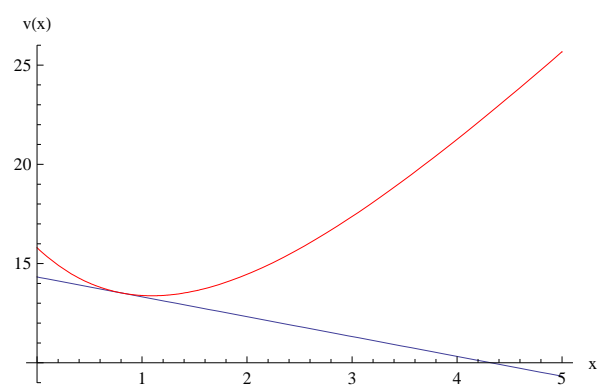

(b)

Figure 1: With parameters $(a, b, \alpha, \lambda, k)=(1,-1,0.2,1.2,0.1)$ : (a) In the transformed space, $H(y)$ and its concave majorant, the horizontal line with height $p=15.797$. (b) In the original space, the value function with the quitting threshold $x^{*}=0.8062$ and the new-job-taking threshold $u^{*}=14.324$. The red line (above) is $v_{0}(x)$ and the blue line (below) is $b x+u^{*}=-x+14.324$.

Since our diffusion is a Browninan motion, $c=-\infty$ and $d=\infty$ are both natural boundaries. In this example, $\varphi(x)=e^{-x \sqrt{2 \alpha}}$ and $F(x)=e^{2 x \sqrt{2 \alpha}}$ and $F^{-1}(y)=\frac{\log y}{2 \sqrt{2 \alpha}}$. It is clear that $l_{-\infty}=0$. We have the explicit form of

$$
H_{0}(y)=\sqrt{y}\left(\frac{b-\frac{a}{\alpha}}{2 \sqrt{2 \alpha}} \cdot \log y+u^{*}\right) .
$$

Note that $\lim _{y \rightarrow 0} H_{0}(y)=0=l_{-\infty}$. By direct computation, we can show that $H_{0}(y)$ satisfies the conditions in the proposition. The concave majorant (see (a)) is just first $H(y)$ itself up to, say $y^{*}$ at which the horizontal line and $H(y)$ match, and thereafter the horizontal line, say $W(F(x))=p$. Recall that $y^{*}=F\left(x^{*}\right)$. After transforming back, it is $p \varphi(x)$. We should add back $g(x)$ to get $v(x)$. Hence our solution is

$$
v(x)= \begin{cases}b x+u^{*}, & x \leq x^{*} \\ v_{0}(x):=p \varphi(x)+\frac{a}{\alpha} x, & x>x^{*}\end{cases}
$$

In the first region where $x<x^{*}$ the agent immediately quit the current job by paying the hurdle cost $b x$ and wait until an offer greater than $u^{*}$ whose expected discounted value is also $u^{*}$ by Lemma 2.4. The value function is the black line (see(b)) in this region. In the second region, the agent should maintain the current job until the Brownian motion hits $x^{*}$, and the value function is the red line.

\subsection{Solution to the second problem}

Let us move on to the second problem (P2) in (2.5) with (2.6). Suppose that we implement the threshold strategy with $u \geq 0$ for the choice of $Y$ as in the first problem. (This premise will be justified in Lemma 2.5.) By setting

$$
J^{\zeta, \tau}(x):=\mathbb{E}^{x}\left[\int_{0}^{\zeta} e^{-\alpha s} f\left(X_{s}\right) d s+e^{-\alpha \zeta} k\left(X_{\zeta}\right)-\int_{0}^{\tau} c e^{-\alpha s} d s+e^{-\alpha \tau} Y_{\tau}\right]
$$

the first two terms of $J$ become

$$
\mathbb{E}^{x}\left[\int_{0}^{\zeta} e^{-\alpha s} f\left(X_{s}\right) d s+e^{-\alpha \zeta} k\left(X_{\zeta}\right)\right]=g(x)+\mathbb{E}^{x}\left[e^{-\alpha \zeta}\left(k\left(X_{\zeta}\right)-g\left(X_{\zeta}\right)\right)\right]
$$


for any stopping time $\zeta \in \mathcal{S}$. Let us denote for $u \geq 0$,

$$
\bar{k}(x):=k(x)-g(x) \quad \text { and } \quad F(d t):=\lambda \bar{G}(u) e^{-\lambda \bar{G}(u) t} d t:=\lambda^{\prime} e^{-\lambda^{\prime} t} d t
$$

where the latter is the arrival time distribution of the thinned Poisson process with $\lambda^{\prime}:=\lambda \bar{G}(u)$. Let us use the fact that, for any $\tau, \zeta \in \mathcal{S}_{2}$,

$$
\zeta \wedge \tau=\zeta 1_{\{\zeta \leq \tau\}}+\tau 1_{\{\zeta>\tau\}}=\zeta 1_{\{\zeta \leq \tau\}}=\zeta .
$$

We then obtain, by conditioning on $\tau$,

$$
\mathbb{E}^{x}\left[e^{-\alpha \zeta}\left(k\left(X_{\zeta}\right)-g\left(X_{\zeta}\right)\right)\right]=\mathbb{E}^{x}\left[e^{-\alpha(\zeta \wedge \tau)} \bar{k}\left(X_{\zeta \wedge \tau}\right)\right]=\mathbb{E}^{x}\left[\int_{0}^{\zeta} e^{-\alpha t} \bar{k}\left(X_{t}\right) F(d t)+\int_{\zeta}^{\infty} e^{-\alpha \zeta} \bar{k}\left(X_{\zeta}\right) F(d t)\right]
$$

due to the independence of the two processes $X$ and $Y$. Then it becomes, by defining

$$
\overline{h^{u}}(x):=\mathbb{E}^{x}\left[\int_{0}^{\infty} \lambda^{\prime} e^{-\left(\alpha+\lambda^{\prime}\right) t} \bar{k}\left(X_{t}\right) d t\right]
$$

and invoking the strong Markov property of $X$ as in (P1),

$$
\begin{aligned}
\mathbb{E}^{x}\left[e^{-\alpha(\zeta \wedge \tau)} \bar{k}\left(X_{\zeta \wedge \tau}\right)\right] & =\bar{h}^{u}(x)-\mathbb{E}^{x}\left[e^{-\left(\alpha+\lambda^{\prime}\right) \zeta} \bar{h}^{u}\left(X_{\zeta}\right)\right]+\mathbb{E}^{x}\left[e^{-\alpha \zeta} \bar{k}\left(X_{\zeta}\right) \int_{\zeta}^{\infty} F(d t)\right] \\
& =\bar{h}^{u}(x)+\mathbb{E}^{x}\left[e^{-\left(\alpha+\lambda^{\prime}\right) \zeta}\left(\bar{k}\left(X_{\zeta}\right)-\bar{h}^{u}\left(X_{\zeta}\right)\right)\right] .
\end{aligned}
$$

Writing down everything, we have for any $u \geq 0$,

$$
\begin{gathered}
J^{\zeta, \tau}(x)=g(x)+\bar{h}^{u}(x)+\mathbb{E}^{x}\left[e^{-\left(\alpha+\lambda^{\prime}\right) \zeta}\left(k\left(X_{\zeta}\right)-g\left(X_{\zeta}\right)-\bar{h}^{u}\left(X_{\zeta}\right)\right)\right] \\
-\left(\frac{c}{\alpha}-\frac{c \lambda^{\prime}}{\alpha\left(\alpha+\lambda^{\prime}\right)}\right)+\frac{\lambda}{\alpha+\lambda^{\prime}} \int_{u}^{\infty} y G(d y) .
\end{gathered}
$$

Notice that the dependence of $\bar{h}^{u}$ on $u$ comes solely from $\lambda^{\prime}=\lambda \bar{G}(u)$. In summary, the second problem (P2) reduces to

$$
\begin{aligned}
\bar{v}(x): & =v(x)-g(x) \\
& =\sup _{\zeta \in \mathcal{S}, u \in R_{+}} \mathbb{E}^{x}\left[e^{-\left(\alpha+\lambda^{\prime}\right) \zeta}\left(k\left(X_{\zeta}\right)-g\left(X_{\zeta}\right)-\bar{h}^{u}\left(X_{\zeta}\right)\right)+\bar{h}^{u}(x)+\frac{1}{\alpha+\lambda^{\prime}}\left(-c+\lambda \int_{u}^{\infty} y G(d y)\right)\right] .
\end{aligned}
$$

Hence we can no longer split the problem into two subproblems. It is intuitively clear that the optimal threshold level for $Y$ depends on the current state $X_{0}=x$ since the agent can start her search before quitting the current one.

Lemma 2.5. For the second problem (P2), the threshold strategy for $Y$ is optimal.

Proof. Recall that $Y_{\tau}$ appears only in the last term of (2.26). The argument is similar to the auxiliary problem $\left(P^{\prime} 1\right)$ and we only need to prove the "closedness" of set $B$. Let us consider case (1) where by the time offer in the amount of $y$ is at table, the agent has not quitted yet or has not picked any offer yet. The corresponding one-step look-ahead is

$$
\max \left(y, \mathbb{E}^{x}\left[\int_{0}^{\zeta \wedge \tau^{(1)}} e^{-\alpha s} f\left(X_{s}\right) d s+e^{-\alpha\left(\zeta \wedge \tau^{(1)}\right)} k\left(X_{\zeta \wedge \tau^{(1)}}\right)-\int_{0}^{\tau^{(1)}} c e^{-\alpha s} d s+e^{-\alpha \tau^{(1)}} Y_{\tau^{(1)}}\right]\right)
$$


where $\tau^{(1)}$, again, is the first jump time of the Poisson process (after $y$ ). We can consider set $B$ similarly to (2.12). It is a mere comparison of the two items in the max function. But $Y$ shows up only at the last term. This observation corresponds to the dependence of $h$ on $u$ only through $\lambda \bar{G}(u)$ in (2.30). Call the first three terms in the expectation $A\left(x, \tau^{(1)}, \zeta\right)$. Similar to Lemma 2.3, we compute

$$
B=\left\{i \in \mathbb{R}_{+}: \frac{\alpha}{\lambda} i \geq \mathbb{E}\left[(Y-i)^{+}\right]+\frac{A\left(x, \tau^{(1)}, \zeta\right)(\alpha+\lambda)}{\lambda}\right\} .
$$

Two cases are possible:

1. While the agent waits for the next offer, she quits. $A\left(x, \tau^{(1)}, \zeta\right)=A(x, \zeta)$

2. While the agent waits for the next offer, she does not quit. $A\left(x, \tau^{(1)}, \zeta\right)=A\left(x, \tau^{(1)}\right)$.

We again allow her to recall the past offers and she stops at $\tau^{(1)}$ with the best offer up to the next arrival of the Poisson process. In either case, the "closedness" of set $B$ is not affected since $A\left(x, \tau^{(1)}, \zeta\right)$ is constant in $i$. Hence $B$ is again a closed set in $i$ for any $\zeta$ and $\tau^{(1)}$ and we invoke Lemma 1.1 to conclude that the first time the process $Y$ enters into this set $B$ is the stopping time.

Finally, we consider case (2) where by the time $y$ is at table, she had already quitted. Then the problem reduces to the first problem (P1). An optimal strategy is characterized already. To conclude, the threshold strategy is again optimal.

Remark 2.3. When we evaluate (2.26) at $\zeta=0$ (the immediate quitting), it becomes

$$
J^{0, \tau}(x)=\mathbb{E}\left[k(x)-\int_{0}^{\tau} c e^{-\alpha s} d s+e^{-\alpha \tau} Y_{\tau}\right],
$$

of which derivative with respect to $u$ is independent of $x$. Taking the derivative with respect to $u$, the first order condition of optimality must become independent of $x$.

Let us again consider an example with the same setting as in Example 2.1 to illustrate the optimization procedure for (P2).

Example 2.2. The setup of the problem is exactly the same as in Example 2.1 except for $\mathcal{S}_{1}$ being replaced by $\mathcal{S}_{2}$. For the second problem (P2), we proceed with (2.30) by computing the necessary functions. Recall $\lambda^{\prime}=\lambda(1-G(u))=\lambda e^{-m u}$ (with $m$ being the rate of Poisson arrival times) is a function of $u$, the offer-taking threshold level.

$$
\begin{aligned}
\bar{k}(x) & =k(x)-g(x)=\left(b-\frac{a}{\alpha}\right) x \\
\bar{h}^{u}(x) & =\mathbb{E}^{x}\left[\int_{0}^{\infty} \lambda^{\prime} e^{-\left(\alpha+\lambda^{\prime}\right) t}\left(b-\frac{a}{\alpha}\right)\left(X_{t}\right) d t\right]=\frac{\lambda^{\prime}}{\alpha+\lambda^{\prime}}\left(b-\frac{a}{\alpha}\right) x .
\end{aligned}
$$

Now let us consider (2.30) by writing everything explicitly:

$$
\begin{aligned}
\bar{v}(x) & =v(x)-g(x) \\
& =\sup _{\zeta \in \mathcal{S}, u \in R_{+}} \mathbb{E}^{x}\left[e^{-\left(\alpha+\lambda^{\prime}(u)\right) \zeta}\left(k\left(X_{\zeta}\right)-g\left(X_{\zeta}\right)-\bar{h}^{u}\left(X_{\zeta}\right)\right)+\bar{h}^{u}(x)+\frac{\lambda}{\alpha+\lambda^{\prime}} \int_{u}^{\infty} y G(d y)\right] \\
& =\sup _{\zeta \in \mathcal{S}, u \in R_{+}} \mathbb{E}^{x}\left[e^{-\left(\alpha+\lambda^{\prime}\right) \zeta}\left(\frac{\alpha\left(b-\frac{a}{\alpha}\right)}{\alpha+\lambda^{\prime}}\right) X_{\zeta}+\frac{\lambda^{\prime}}{\alpha+\lambda^{\prime}}\left(b-\frac{a}{\alpha}\right) x+\frac{\lambda}{\alpha+\lambda^{\prime}} \int_{u}^{\infty} y G(d y)\right] \\
& =\sup _{\zeta \in \mathcal{S}, u \in R_{+}} \mathbb{E}^{x}\left[e^{-\left(\alpha+\lambda^{\prime}\right) \zeta}\left(\frac{\alpha\left(b-\frac{a}{\alpha}\right)}{\alpha+\lambda^{\prime}}\right) X_{\zeta}+\frac{\lambda^{\prime}}{\alpha+\lambda^{\prime}}\left(\left(b-\frac{a}{\alpha}\right) x+u+\frac{1}{m}\right)\right] .
\end{aligned}
$$


We can first solve the optimal stopping problem for a fixed $u$ and find the optimal threshold for quitting in terms of $u$. Namely we solve, for a given $u$,

$$
\sup _{\zeta \in \mathcal{S}} \mathbb{E}^{x}\left[e^{-\left(\alpha+\lambda^{\prime}\right) \zeta}\left(\frac{\alpha\left(b-\frac{a}{\alpha}\right)}{\alpha+\lambda^{\prime}}\right) X_{\zeta}\right] .
$$

Again suppressing the dependence of $\lambda^{\prime}$ on $u$, we find, similarly to the first problem, $\varphi(x)=e^{-x \sqrt{2\left(\alpha+\lambda^{\prime}\right)}}$ and $F(x)=e^{2 x \sqrt{2\left(\alpha+\lambda^{\prime}\right)}}$ with $F^{-1}(y)=\frac{\log y}{2 \sqrt{2\left(\alpha+\lambda^{\prime}\right)}}$. After the transformation, the reward function $\bar{h}^{u}(\cdot)$ is

$$
H^{u}(y):=\frac{\alpha\left(b-\frac{a}{\alpha}\right)}{\left(\alpha+\lambda^{\prime}\right) 2 \sqrt{2\left(\alpha+\lambda^{\prime}\right)}} \log y \sqrt{y} .
$$

This function attains the maximum at $y_{0}=e^{-2}$ independent of $u$. The smallest concave majorant of $H^{u}(y)$ is again the horizontal line that matches $H^{u}(y)$ at $y_{0}=e^{-2}$ for all $u$. But in the original space, the quitting threshold for $u, D(u):=F^{-1}\left(y_{0}\right)=\frac{\log y_{0}}{2 \sqrt{2\left(\alpha+\lambda^{\prime}\right)}}$ depends on $u$. See the picture below for $u=10$.

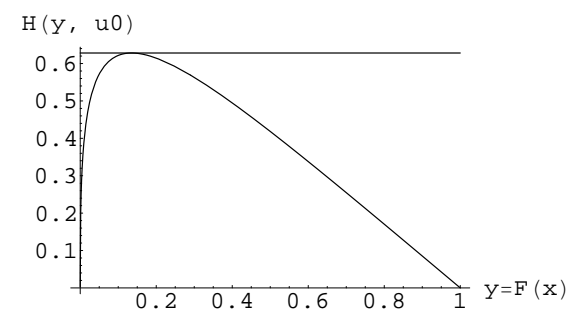

(a)

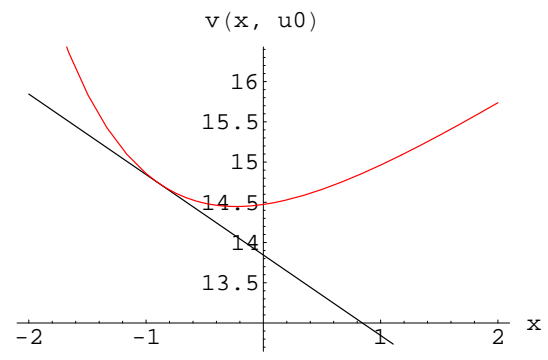

(b)

Figure 2: With parameters $(a, b, \alpha, \lambda, m)=(1,-1,0.2,1.2,0.1)$ : (a) In the transformed space, $H^{u}(y)$ with $u=u_{0}=10$ and its concave majorant. (b) In the original space, the value function with the quitting threshold $x\left(u_{0}\right)=-0.892728$. The red line (above) is $v_{0}\left(x ; u_{0}\right)$ and the black line (below) is the reward function

Graph (c) is the plot of the threshold level for quitting $D(u)$ for various $u$ 's. Now the intercept (=the height of

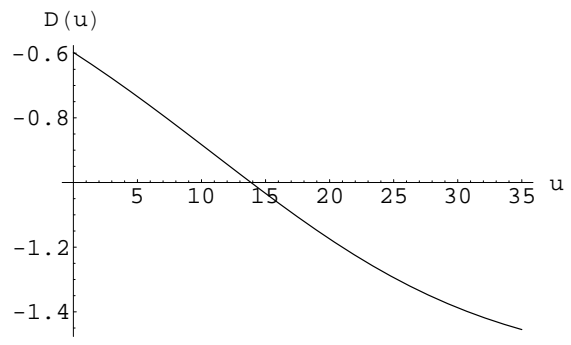

(c)

Figure 3: The optimal quitting threshold $D(u)$ for various $u$ 's.

the horizontal line) is

$$
p(u):=H^{u}\left(y_{0}\right)=\frac{\alpha\left(b-\frac{a}{\alpha}\right)}{\left(\alpha+\lambda^{\prime}\right) 2 \sqrt{2\left(\alpha+\lambda^{\prime}\right)}}\left(\frac{-2}{e}\right) .
$$


Hence the value function for any $u \geq 0$ is written of the form

$$
v^{u}(x):= \begin{cases}\left(\frac{\alpha\left(b-\frac{a}{\alpha}\right)}{\alpha+\lambda^{\prime}}\right) x+\frac{\lambda^{\prime}}{\alpha+\lambda^{\prime}}\left(\left(b-\frac{a}{\alpha}\right) x+u+\frac{1}{m}\right)+\frac{a}{\alpha} x, & x<D(u), \\ v_{0}(x, u):=p(u) e^{-x \sqrt{2\left(\alpha+\lambda^{\prime}\right)}+\frac{\lambda^{\prime}}{\alpha+\lambda^{\prime}}\left(\left(b-\frac{a}{\alpha}\right) x+u+\frac{1}{m}\right)+\frac{a}{\alpha} x,} & x \geq D(u) .\end{cases}
$$

It can be simplified but let us keep it for the moment. Finally, we maximize $v^{u}(x)$ with respect to $u$ by simply taking the partial derivative with respect to $u$. Let us first consider the second branch in (2.33), that is, $v_{0}(x, u)$ to find the optimal offer-taking threshold level, say $u_{x}$ for each initial state $x$. We can directly show that, for each $x$, the function $v_{0}(x, u)$ attains the sole local maximum. See graph (d) below. In graph (e), we also plot the threshold level $x \rightarrow u_{x}$ for each initial state $x$. It is increasing in $x$. This makes sense since if the initial position is high, then the offer-taking level should also be high.

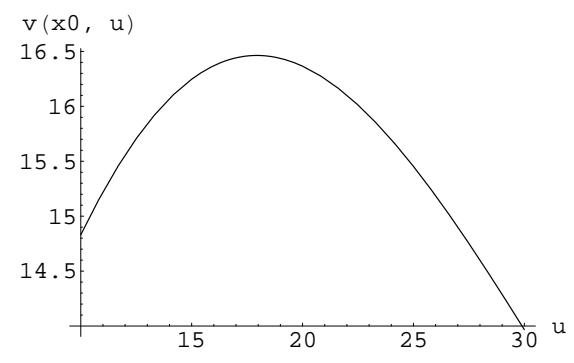

(d)

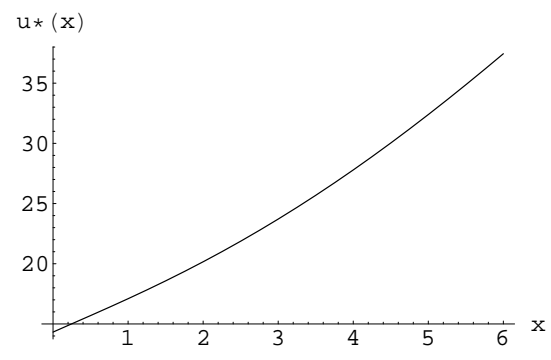

(e)

Figure 4: With parameters $(a, b, \alpha, \lambda, m)=(1,-1,0.2,1.2,0.1)$ : (a) For given $x_{0}, v_{0}\left(x_{0} ; u\right)$ attains the sole maximum. (b) The optimal threshold level of taking an offer $u_{x}$ as a function of the initial state $x$.

The final question is the following: Suppose the agent's current state in the current job is at $x$. She computes the best offer-taking level, say $u_{x}$ by taking the derivative of the second branch of $v^{u}(x)$, namely $v_{0}(x, u)$. Does this $u_{x}$ also maximizes the other function of $v^{u}(x)$, that is, the first branch in (2.33)?

To answer this question, we simplified the first branch of (2.33) to get

$$
v^{u}(x)=b x+\frac{\lambda^{\prime}}{\alpha+\lambda^{\prime}}\left(u+\frac{1}{m}\right), \quad x<D(u) .
$$

The maximizer of $u$ is independent of $x$ (see also Remark 2.3) and is the solution of

$$
\frac{e^{-m u}}{m}=\frac{\alpha u}{\lambda}
$$

which is exactly the same equation as (2.23) in the first example for the universally best $u^{*}$. So if we plug $u^{*}$ back into (2.34), it becomes

$$
v^{u}(x)=b x+u^{*}, \quad x<D(u) .
$$

This is the same function as in the first problem (See (2.25)). Of course, this is not a coincidence. We will generalize this later. This observation makes sense because this function represents the value of an immediate quitting. Hence this function has to be the same both in the first and the second problem. We summarize the optimal procedure:

1. Given the initial state $x$, she maximizes the second branch of $v^{u}(x)$ in (2.33) to get the offer-taking threshold $u_{x}$ as in graph (d). 
2. Based on this $u_{x}$, she computes the optimal quitting threshold of $D\left(u_{x}\right)$ by solving (2.32).

3. Compare this $D\left(u_{x}\right)$ with the initial $x$ if $x \leq D\left(u_{x}\right)$, quit immediately and get $b x+u^{*}$. Otherwise, stay with the current job and her value function is $v_{0}\left(x, u_{x}\right)$ in (2.33).

4. If she chooses to stay with the current job, two possibilities are there: If the diffusion hits $D\left(u_{x}\right)$ first, quit and wait for a new one with higher value than $u_{x}$. If a new offer shows up (with a value higher than $u_{x}$ ) before the hitting time of the diffusion, quit and take the new job at the same time.

Therefore, the answer to the question at the top of this paragraph is as follows: The maximizer of the second branch, $u_{x}$ is in general not the same as $u^{*}$, the maximizer of the first branch. But the agent does not have to worry. Once $D\left(u_{x}\right)$ is computed and turns out to be greater than $x$, her choice is immediate quitting. At this point, her immediate quitting is guaranteed to be optimal irrespective of $x$ because $u^{*}$ is independent of $x$.

Again, while this method can handle all the possible selections of $f, k, G$ and $X$, we shall specify sufficient conditions for computational convenience. Let us define the increasing and decreasing solutions of $(\mathcal{A}-(\alpha+$ $\lambda \bar{G}(u)) v(x)=0$ by $\psi(x)$ and $\varphi(x)$ with a slight abuse of notation. We define $F(x)$ accordingly. Recall $\bar{h}^{u}(\cdot)$ is defined in (2.28). With these definitions, we summarize the result as follows:

Proposition 2.2. Suppose that the state space $(c, d) \subseteq \mathbb{R}$ of $X$ has natural boundaries at $c$ and $d$ with $l_{c}<\infty$. If $H_{0}(y):=\bar{h}^{u_{x}}\left(F^{-1}(y)\right) / \varphi\left(F^{-1}(y)\right)$ has sole local maximum in the interior of $(F(c), F(d))$ with $\lim _{y \rightarrow c} H_{0}^{\prime \prime}(y)<$ 0 and $\lim _{y \rightarrow d} H_{0}^{\prime \prime}(y)>0$, then the value function, that is the solution to (P2) in (2.3), is of the form

$$
v(x)= \begin{cases}k(x)+u^{*}, & x<D\left(u_{x}\right), \\ p\left(u_{x}\right) \varphi(x)+\bar{h}^{u_{x}}(x)+g(x), & x \geq D\left(u_{x}\right),\end{cases}
$$

where

$$
u_{x}:=\arg \max _{u \in \mathbb{R}_{+}}\left(p(u) \varphi(x)+\bar{h}^{u}(x)\right)
$$

for any given $x$. Here $u^{*}$ (independent of $x$ ) is the unique solution of (2.15). $p\left(u_{x}\right)$ and $D\left(u_{x}\right)$ are uniquely determined.

If $x<D\left(u_{x}\right)$, then the optimal strategy is to quit the current job immediately and to wait for a first job offer whose value is greater than or equal to $u^{*}$. If $x \geq D\left(u_{x}\right)$, then the optimal strategy is to wait until $\zeta \wedge \tau$ where $\zeta=\inf \left\{t>0: X_{t} \leq D\left(u_{x}\right)\right\}$ and $\tau=\inf \left\{t>0: Y_{t} \geq u_{x}\right\}$.

Proof. Evaluating (2.30) at $\zeta=0$, we have

$$
v(x)=k(x)+\sup _{u \in R_{+}}\left\{\frac{1}{\alpha+\lambda^{\prime}}\left(-c+\lambda \int_{u}^{\infty} y G(d y)\right)\right\} .
$$

for $x<D(u)$. Working out in the same way as in Lemma 2.3, we can find that the optimal $u^{*}$ satisfies (2.15) and the previous equation becomes

$$
v(x)=k(x)+u^{*}, \quad x<D(u)
$$

Note that $u^{*}$ is independent of $D(u)$. For the other branch of $v(x)$, the proof is similar to Proposition 2.1. 


\section{Search problem in a jump diffusion model}

${ }^{1}$ In this section, we extend our model by adding stochastic jumps in the state space of process $X$. Let $\{\Omega, \mathcal{F}, \mathbb{P}\}$ be a probability space hosting a Brownian motion $B$ and an independent Poisson random measure $M(d t, d z)$ on $[0, \infty) \times \mathbb{R}$, both adapted to a certain filtration $\mathbb{F}$ that satisfies the usual conditions. We assume that this Poisson random measure is independent of the Poisson process $N=\{N(t) ; t \geq 0\}$ associated with arrival times of job offers $Y$ in the previous sections. The mean measure of $M$ is $\nu(d t, d z)=\theta d t F(d z)$, where $\theta>0$ is constant and $F(d z)$ is the common distribution of jump sizes. Throughout this subsection, we consider

$$
X_{t}=X_{0}+\mu t+\sigma B_{t}-\sum_{i=1}^{M(t)} Z_{i}, \quad X_{0}=x,
$$

where $M=\{M(t): t \geq 0\}$ is a Poisson process (associated with the Poisson random measure $M(d t, d z)$ ) with constant intensity rate $\theta$ and jump sizes $Z_{i}, i=1,2,3 \ldots$ are i.i.d. from an exponential distribution with parameter $\eta$. Hence our mean measure becomes $\nu(d t, d z)=\theta d t \eta e^{-\eta z} d z$ and the jumps are always downwards. These negative jumps represent a sudden drop of the agent's incentives. For modeling purposes, these downward jumps may be compensated by setting the drift parameter $\mu$ higher. The infinitesimal generator of this process acting on a test function $u \in C^{2}$ is

$$
\mathcal{A} u(x)=\frac{1}{2} \sigma^{2} u^{\prime \prime}(x)+\mu u^{\prime}(x)+\theta \int_{0}^{\infty}(u(x-z)-u(x)) \eta e^{-\eta z} d z .
$$

Our jump diffusion setup in the state variable (current job) does not change the structure of problems (P1) or (P2) at all. Hence our presentation here focus on the solution of the optimal stopping problem in (2.19) and we provide an explicit solution in the context of Example 2.1. Of course, the optimal stopping part (by fixing $u$ value) in (2.30) is very similar. Here we use a different approach from the ordinary Hamilton-Jacobi-Bellman type. Due to the memoryless property of exponential distribution (of the jump size), we can directly evaluate the functional associated with the exit time from an interval, the value of a certain function of $X$ at which the process $X$ cross the left boundary of $(a, \infty)$. Using this functional, we can identify the form of the value function (see (3.14)), while the optimality is still proved by a verification lemma. But we do not have to set the boundary conditions to determine the candidate of the value function. Hence this method facilitates the proof of the existence and uniqueness of the system of non-linear equations that arise from the boundary conditions. This analysis is along the line of Kou and Wang [13], and this is one of the few examples that an explicit solution to an optimal stopping problem in an jump diffusion model. Other direct methods successful in obtaining explicit solutions (in terms of the increasing minimal harmonic map) in a spectrally negative jump-diffusion model include Alvarez and Rakkolainen [1, 2, 3] where they solve various types control problems including optimal stopping, impulse control and singular stochastic control. The well-known alternative technique is based on factorization arguments. Mordecki and Salminen [15] show the value function for optimal stopping problems driven by general Hunt processes as an integral representation of excessive functions. See also Boyarchenko and Levendorskii [7, 8] and references therein for this approach.

\subsection{Exit problem from an interval}

If we try a function in the form of $u(x)=e^{\beta x}$ with $\beta+\eta>0$ for $(\mathcal{A}-\alpha) u(x)=0$, then $\beta$ 's are found by the roots of the following equation:

$$
G(\beta):=\frac{1}{2} \sigma^{2} \beta^{2}+\mu \beta-\frac{\theta \beta}{\eta+\beta}=\alpha .
$$

\footnotetext{
${ }^{1}$ For this section, we thank Savas Dayanik for valuable discussions.
} 
Now, by the independence of $B_{t}, M_{t}$ and $Z_{i}$,

$$
\begin{aligned}
\mathbb{E}^{x}\left[e^{r X_{t}}\right] & =\mathbb{E}^{x}\left[e^{r\left(X_{0}+\mu t+\sigma B_{t}\right)} \prod_{i=1}^{M_{t}} e^{-r Z_{i}}\right]=e^{r x} e^{r \mu t+\frac{1}{2} \sigma^{2} r^{2} t} \mathbb{E}\left[\left(\frac{\eta}{\eta+r}\right)^{M_{t}}\right]=e^{r x} e^{t\left(r \mu+\frac{1}{2} \sigma^{2} r^{2}+\theta\left(\frac{\eta}{\eta+r}-1\right)\right)} \\
& =e^{r x} e^{G(r) t}
\end{aligned}
$$

Hence we have $\mathbb{E}^{x}\left[e^{r X_{t}-G(r) t}\right]=1$, which implies that $\left\{\exp \left(r X_{t}-G(r) t\right)\right\}$ is a martingale for any $r \in \mathbb{R}$. Equation (3.3) has at most three real roots, two negative roots $-\beta_{1}$ and $-\beta_{2}$, and one positive root $\beta_{3}$, with the relationship

$$
-\beta_{2}<-\eta<-\beta_{1}<0 \quad \text { and } \quad \beta_{3}>0
$$

Namely,

$$
0<\beta_{1}<\eta<\beta_{2} \quad \text { and } \quad \beta_{3}>0
$$

Let us compute some functionals of $X$ associated with exit time from an interval $[a, b] \in \mathbb{R}$. Define $T_{a} \triangleq \inf \{t \geq$ $\left.0, X_{t} \leq a\right\}$ and note that due to the negativity of the jumps, $X_{T_{a}} \leq a$. Note that we have $\mathbb{P}^{x}\left(T_{a}<\infty\right)=1$ for all $a$ and $x$ such that $-\infty<a<x<\infty$.

Lemma 3.1. Suppose that we have a finite interval $[a, b] \in \mathbb{R}$ and a process that is defined by (3.1) with mean measure $\nu(d t, d z)=\theta d t \eta e^{-\eta z} d z$. Then, for $a \leq x \leq b$, we have

$$
\text { (6) } \quad \mathbb{E}^{x}\left[e^{-\alpha T_{b}} \mathbf{1}_{\left\{T_{a}>T_{b}\right\}}\right]=\frac{C(x)}{A} e^{\beta_{3}(x-b)} \text {. }
$$

Furthermore, for every $x<b, \lim _{a \downarrow-\infty} \mathbb{E}^{x}\left[e^{-\alpha T_{b}} \mathbf{1}_{\left\{T_{a}>T_{b}\right\}}\right]=e^{\beta_{3}(x-b)}$ where

$$
\begin{aligned}
A & =\frac{\eta}{\eta+\beta_{3}}\left(\frac{\beta_{1}+\beta_{3}}{\eta-\beta_{1}} \varphi_{1}(a)\left(\varphi_{2}(a)-\varphi_{2}(b)\right)-\frac{\beta_{2}+\beta_{3}}{\eta-\beta_{2}} \varphi_{2}(a)\left(\varphi_{1}(a)-\varphi_{1}(b)\right)\right), \\
B_{1}(x) & =\left(\varphi_{2}(a)-\varphi_{2}(b)\right)\left(\varphi_{1}(x)-\varphi_{1}(b)\right)-\left(\varphi_{1}(a)-\varphi_{1}(b)\right)\left(\varphi_{2}(x)-\varphi_{2}(b)\right), \\
B_{2}(x) & =-\left(\frac{\eta}{\eta-\beta_{2}} \varphi_{2}(a)-\frac{\eta}{\eta+\beta_{3}} \varphi_{2}(b)\right)\left(\varphi_{1}(x)-\varphi_{1}(b)\right)+\left(\frac{\eta}{\eta-\beta_{1}} \varphi_{1}(a)-\frac{\eta}{\eta+\beta_{3}} \varphi_{1}(b)\right)\left(\varphi_{2}(x)-\varphi_{2}(b)\right), \\
C(x) & =\frac{\eta}{\eta+\beta_{3}}\left(\frac{\beta_{1}+\beta_{3}}{\eta-\beta_{1}} \varphi_{1}(a)\left(\varphi_{2}(a)-\varphi_{2}(x)\right)+\frac{\beta_{2}+\beta_{3}}{\eta-\beta_{2}} \varphi_{2}(a)\left(\varphi_{1}(x)-\varphi_{1}(a)\right)\right), \\
\text { with } \varphi_{1}(\cdot) & =e^{-\left(\beta_{1}+\beta_{3}\right)(\cdot)} \text { and } \varphi_{2}(\cdot)=e^{-\left(\beta_{2}+\beta_{3}\right)(\cdot)} .
\end{aligned}
$$

Proof. For (1), we need to compute $v(x):=\mathbb{E}^{x}\left[e^{-\alpha T_{a}}\right]$. Let $u(x)$ be the bounded continuous solution of $(\mathcal{A}-$ $\alpha) u=0$ on $(a, \infty)$ and $u(x)=1$ on $(-\infty, a]$. Then $u(x)=A_{1} e^{-\beta_{1} x}+A_{2} e^{-\beta_{2} x}+A_{3} e^{\beta_{3} x}$. Since we want $u(x)$ 
to be bounded, we must set $A_{3}=0$. Note that while $e^{-\beta_{1} x}$ is a martingale function, $e^{-\beta_{2} x}$ is not a martingale function because of $\beta_{2}>\eta$. Indeed, $\mathcal{A} e^{-\beta_{2} x}=+\infty \neq \alpha e^{-\beta_{2} x}$, even though $-\beta_{2}$ satisfies equation (3.3). Nevertheless, $\beta_{2}$ plays an important role below. We proceed to determine the coefficients $A_{1}$ and $A_{2}$. First, by expanding $(\mathcal{A}-\alpha) u(x)$ with (3.3), we have

$$
\begin{aligned}
(\mathcal{A}-\alpha) u(x) & =\frac{1}{2} \sigma^{2} u^{\prime \prime}(x)+\mu u^{\prime}(x)+\theta \int_{0}^{x-a}(u(x-y)-u(x)) F(d y)+\theta \int_{x-a}^{\infty}(1-u(x)) F(d y)-\alpha u(x) \\
& =\theta e^{-\eta(x-a)}-\frac{\theta \eta}{\eta-\beta_{1}} A_{1} e^{-x \eta+a\left(\eta-\beta_{1}\right)}-\frac{\theta \eta}{\eta-\beta_{2}} A_{2} e^{-x \eta+a\left(\eta-\beta_{2}\right)} .
\end{aligned}
$$

Setting $(\mathcal{A}-\alpha) u(x)=0$, we have a condition for $A_{1}$ and $A_{2}$,

$$
1=\frac{\theta \eta}{\eta-\beta_{1}} A_{1} e^{-a \beta_{1}}-\frac{\theta \eta}{\eta-\beta_{2}} A_{2} e^{-a \beta_{2}}
$$

with the continuity condition, that is, $u(a+)=1$,

$$
1=A_{1} e^{-a \beta_{1}}+A_{2} e^{-a \beta_{2}}
$$

By solving (3.11) and (3.12), we have $A_{1}=\frac{\beta_{2}\left(\eta-\beta_{1}\right)}{\eta\left(\beta_{2}-\beta_{1}\right)} e^{\beta_{1} a}$, and $A_{2}=\frac{\beta_{1}\left(\eta-\beta_{2}\right)}{\eta\left(\beta_{2}-\beta_{1}\right)} e^{\beta_{2} a}$. Hence we have

$$
u(x)= \begin{cases}1, & x \leq a \\ \frac{\beta_{2}\left(\eta-\beta_{1}\right)}{\eta\left(\beta_{2}-\beta_{1}\right)} e^{-\beta_{1}(x-a)}+\frac{\beta_{1}\left(\beta_{2}-\eta\right)}{\eta\left(\beta_{2}-\beta_{1}\right)} e^{-\beta_{2}(x-a)}, & x>a .\end{cases}
$$

For the proof of $v(x)=u(x)$, we refer the reader to Kou and Wang [13] (Theorem 3.1) ${ }^{2}$. The proof of the other functionals are similar. For example, for equation (4), define

$$
w_{1}(x):= \begin{cases}1, & x \leq a-z, \\ 0, & x \in[a-z, a] \cup[b, \infty), \\ A_{1} e^{-\beta_{1} x}+A_{2} e^{-\beta_{2} x}+A_{3} e^{\beta_{3} x}, & a<x<b .\end{cases}
$$

Three conditions to determine $A_{1}, A_{2}$, and $A_{3}$ come from $w_{1}(a+)=0, w_{1}(b-)=0$, and the martingale condition $(\mathcal{A}-\alpha) w_{1}(x)=0$ for $a<x<b$. Note that, in this case,

$$
\begin{aligned}
(\mathcal{A}-\alpha) w_{1}(x) & =\frac{1}{2} \sigma^{2} w_{1}^{\prime \prime}(x)+\mu w_{1}^{\prime}(x) \\
& +\theta w_{1}(x)-\alpha w_{1}(x)+\theta\left(\int_{0}^{x-a}+\int_{x-a}^{x-a+z}+\int_{x-a+z}^{\infty}\right) w_{1}(x-y) F(d y) d y
\end{aligned}
$$

and

$$
w_{1}(x-y)= \begin{cases}A_{1} e^{-\beta_{1}(x-y)}+A_{2} e^{-\beta_{2}(x-y)}+A_{3} e^{\beta_{3}(x-y)}, & y \in[0, x-a), \\ 0, & y \in[x-a, x-a+z], \\ 1, & y \in[x-a+z, \infty) .\end{cases}
$$

After finding the expressions for $A_{1}, A_{2}$, and $A_{3}$, we can use the same argument as before to find the desired expectation.

Lemma 3.2. (a) Given $x \in(a, \infty) \subseteq \mathbb{R} \cup\{+\infty\}$, and $h: \mathbb{R} \rightarrow \mathbb{R}$, a polynomial of degree $n$, we have

$$
\mathbb{E}^{x}\left[e^{-\alpha T_{a}} h\left(X_{T_{a}}\right)\right]=P(x) \sum_{i=0}^{n} \frac{(-1)^{i} h^{(i)}(a)}{\eta^{i}}+h(a) Q(x) .
$$

\footnotetext{
${ }^{2}$ In this paper, a doubly exponential distribution is studied and results similar to $(1) \sim(3)$ of Lemma 3.1 are available.
} 
where

$$
\begin{aligned}
& P(x)=\frac{\left(\eta-\beta_{1}\right)\left(\beta_{2}-\eta\right)}{\eta\left(\beta_{2}-\beta_{1}\right)}\left(e^{-\beta_{1}(x-a)}-e^{-\beta_{2}(x-a)}\right), \\
& Q(x)=\frac{\eta-\beta_{1}}{\beta_{2}-\beta_{1}} e^{-\beta_{1}(x-a)}+\frac{\beta_{2}-\eta}{\beta_{2}-\beta_{1}} e^{-\beta_{2}(x-a)} .
\end{aligned}
$$

(b) Given $x \in(a, b)$, we have

$$
\mathbb{E}^{x}\left[e^{-\alpha\left(T_{a} \wedge T_{b}\right)} h\left(X_{T_{a} \wedge T_{b}}\right)\right]=\frac{B_{1}(x)}{A} \sum_{i=0}^{n}(-1)^{i} \frac{h^{(i)}(a)}{\eta^{i}} e^{\beta_{3}(x-a)}+\frac{B_{2}(x)}{A} h(a) e^{\beta_{3}(x-a)}+\frac{C(x)}{A} h(b) e^{\beta_{3}(x-b)} .
$$

Proof. (a) Let $\Gamma_{a}$, a non-negative random variable, be the size of undershoot at the left boundary $a$. Then

$$
\begin{aligned}
\mathbb{E}^{x}\left[e^{-\alpha T_{a}} h\left(X_{T_{a}}\right)\right] & =\mathbb{E}^{x}\left[e^{-\alpha T_{a}} h\left(a-\Gamma_{a}\right)\right]=\int_{0}^{\infty} h(a-z) \mathbb{E}^{x}\left[e^{-\alpha T_{a}} 1_{\left\{\Gamma_{a} \in d z\right\}}\right]+h(a) \mathbb{E}^{x}\left[e^{-\alpha T_{a}} \mathbf{1}_{\left\{a-X_{T_{a}}=0\right\}}\right] \\
& =\int_{0}^{\infty} h(a-z) \eta e^{-\eta z} P(x) d z+h(a) Q(x),
\end{aligned}
$$

by using (3.6) and (3.7). Integration by parts in the first term will lead to our result. The proof of $(b)$ is similar.

\subsection{Solution to the optimal stopping problem}

We continue to solve Example 2.1. Namely the optimal stopping part of the problem (cf. (2.24)) is

$$
\begin{aligned}
\bar{v}(x) & =\sup _{\zeta \in \mathcal{S}_{1}} \mathbb{E}^{x}\left[e^{-\alpha \zeta}\left\{\left(b-\frac{a}{\alpha}\right) x+\frac{\lambda}{\alpha+\lambda \bar{G}\left(u^{*}\right)} \int_{u^{*}}^{\infty} y G(d y)\right\}\right] \\
& =\sup _{\zeta \in \mathcal{S}_{1}} \mathbb{E}^{x}\left[e^{-\alpha \zeta}\left\{\left(b-\frac{a}{\alpha}\right) x+u^{*}\right\}\right],
\end{aligned}
$$

where we define $h(x):=(b-a / \alpha) x+u^{*}:=r x+u^{*}$ for notational brevity. Note that $r:=(b-a / \alpha)<0$.

\subsubsection{Necessary condition of the optimality}

By Lemma 3.2, for any interval $[l, \infty)$,

$$
\begin{aligned}
\mathbb{E}^{x}\left[e^{-\alpha T_{l}}\left(r X_{T_{l}}+u^{*}\right)\right] & =\frac{\eta-\beta_{1}}{\eta\left(\beta_{2}-\beta_{1}\right)}\left(\left(\eta-\beta_{2}\right) \frac{r}{\eta}+\beta_{2}\left(r l+u^{*}\right)\right) e^{-\beta_{1}(x-l)} \\
& +\frac{\beta_{2}-\eta}{\eta\left(\beta_{2}-\beta_{1}\right)}\left(\left(\eta-\beta_{1}\right) \frac{r}{\eta}+\beta_{1}\left(r l+u^{*}\right)\right) e^{-\beta_{2}(x-l)} .
\end{aligned}
$$

Hence the optimal stopping problem reduces to finding some $l$ (call it $l^{*}$ ) that maximizes this function for all $x \in \mathbb{R}$. To derive the necessary condition of the optimality of $l^{*}$, we take the derivative with respect to $l$ and evaluate in particular at $x=0$ since $l^{*}$ has to maximize the function for all $x$. After some simple algebra, we obtain

$$
l^{*}=-\left(\frac{\beta_{1}+\beta_{2}}{\beta_{1} \beta_{2}}-\frac{1}{\eta}+\frac{u}{r}\right) .
$$

Plugging (3.15) into the right hand side of (3.14) and set this result as

$$
w_{0}(x):=\frac{-r}{\eta\left(\beta_{2}-\beta_{1}\right)}\left(\frac{\left(\eta-\beta_{1}\right) \beta_{2}}{\beta_{1}} e^{-\beta_{1}\left(x-l^{*}\right)}+\frac{\left(\beta_{2}-\eta\right) \beta_{1}}{\beta_{2}} e^{-\beta_{2}\left(x-l^{*}\right)}\right) .
$$

Note that by direct calculation, $w_{0}\left(l^{*}\right)=-r\left(\frac{1}{\eta}-\frac{\left(\beta_{1}+\beta_{2}\right)}{\beta_{1} \beta_{2}}\right)$, which is equal to $r l^{*}+u^{*}$ in light of (3.15) and also $w_{0}^{\prime}\left(l^{*}\right)=r$ as expected. 


\subsubsection{Proof of sufficiency of the optimality}

The construction of $w_{0}(x)$ suggests us to define

$$
w(x):= \begin{cases}r x+u^{*}, & x \leq l^{*} \\ w_{0}(x), & x>l^{*}\end{cases}
$$

The rest of the task is to prove the optimality of $w(x)$ via variational inequalities. That is, to show $w(x)=\bar{v}(x)$ for all $x \in \mathbb{R}$ by proving

$$
\max (h(x)-w(x),(\mathcal{A}-\alpha) w(x))=0
$$

for all $x \in \mathbb{R}$. For the variational inequality, see standard textbooks, e.g. Øksendal and Sulem [17].

(1) $w(x) \geq r x+u^{*}$ : When $x \leq l^{*}, w(x)-\left(r x+u^{*}\right)=0$. Let us define $D(x):=w_{0}(x)-\left(r x+u^{*}\right)$. Now $D^{\prime}(x)=w_{0}^{\prime}(x)-r$ and $D^{\prime \prime}(x)=w_{0}^{\prime \prime}(x)$. We also know that $D\left(l^{*}\right)=0$ and $D^{\prime}\left(l^{*}\right)=0$. Then we have

$$
D^{\prime \prime}(x)=w_{0}^{\prime \prime}(x)=\frac{\beta_{1} \beta_{2}(-r)}{\eta\left(\beta_{2}-\beta_{1}\right)}\left(\left(\eta-\beta_{1}\right) e^{-\beta_{1}\left(x-l^{*}\right)}+\left(\beta_{2}-\eta\right) e^{-\beta_{2}\left(x-l^{*}\right)}\right)>0, \quad x \in \mathbb{R},
$$

by (3.4), showing that $D^{\prime}(x)$ is increasing everywhere. Since $D^{\prime}\left(l^{*}\right)=0$, it follows that $D^{\prime}(x)>0$ on $\left(l^{*}, \infty\right)$. This fact together with $D\left(l^{*}\right)=0$ implies that $D(x)>0$ for $x>l^{*}$. Therefore, we have established that $w_{0}(x)>r x+u^{*}$ on $\left(l^{*}, \infty\right)$.

(2) $(\mathcal{A}-\alpha) w(x) \leq 0$ : When $x \geq l^{*},(\mathcal{A}-\alpha) w(x)=(\mathcal{A}-\alpha) w_{0}(x)=0$ by the construction of $w_{0}(x)$. Finally, on $x<l^{*}$,

$$
\begin{aligned}
(\mathcal{A}-\alpha) w(x) & =r \mu+\theta \int_{0}^{\infty}\left(r(x-z)+u^{*}-\left(r x+u^{*}\right)\right) \eta e^{-\eta z} d z-\alpha\left(r x+u^{*}\right) \\
& =r \mu-r \theta / \eta-\alpha\left(r x+u^{*}\right) \\
& <r \mu-r \theta / \eta-\alpha\left(r l^{*}+u^{*}\right)=\lim _{x \uparrow l^{*}}(\mathcal{A}-\alpha) w(x) \\
& =\lim _{x \downarrow l^{*}}(\mathcal{A}-\alpha) w(x)-\frac{1}{2} \sigma^{2} w_{0}^{\prime \prime}\left(l^{*}\right)<\lim _{x \downarrow l^{*}}(\mathcal{A}-\alpha) w(x)=0
\end{aligned}
$$

where the last inequality is due to (3.19). This proves the desired variational inequality (3.18). Hence $w(x)=\bar{v}(x)$ and $v(x)=w(x)+g(x)$.

The next picture shows the case with $\theta=0.5$ and $\eta=1$ and the other parameters are the same as in Example 2.1 and 2.2. The quitting threshold level is lower than the no jump case: $l^{*}=-1.17583<0.8062=x^{*}$. It is reasonable to think that negative jumps will make the downfall of the diffusion faster and therefore, the discount factor $e^{-\alpha t}$ does reduce the value as much as the case with no jumps. Hence the agent ends up with a lower threshold.

\section{References}

[1] L. H. R. Alvarez and T. Rakkolainen. A class of solvable optimal stopping problems of spectrally negative jump diffusions. Aboa Centre of Economic Discussion Paper 9, 2006. 


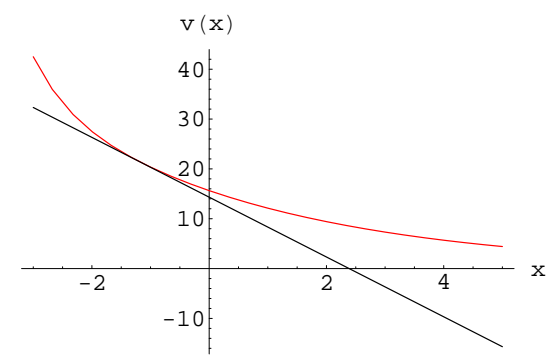

Figure 5: The value function in a jump diffusion model for the first example. On $x<l^{*}, v(x)=r x+u^{*}$ (black line, below) and on $x>l^{*}, v(x)=w_{0}(x)+g(x)$ (red line, above).

[2] L. H. R. Alvarez and T. Rakkolainen. Investment timing in presenxe of downside risk: a certainty equivalent characterization. Annals of Finance, to appear.

[3] L. H. R. Alvarez and T. Rakkolainen. Optimal payout policy in presense of downside risk. Math. Meth. Oper. Res., to appear.

[4] F. A. Boshuizen and J. M. Gouweleeuw. General optimal stopping theorems for semi-markov processes. Adv. Appl. Prob., 25:825-846, 1993.

[5] F. A. Boshuizen and J. M. Gouweleeuw. A general framework for optimal stopping problems associated with multivariate point processes, and applications. Sequential Analysis, 13:351-365, 1994.

[6] F. A. Boshuizen and J. M. Gouweleeuw. A continuous-time job search model: general renewal processes. Stochastic Models, 11:349-369, 1995.

[7] S. I. Boyarchencko and S. Z. Levendorskii. Optimal stopping made easy. J. Math. Econ., 43:201-217, 2002.

[8] S. I. Boyarchencko and S. Z. Levendorskii. Perpetual American options under Lévy processes. SIAM Journal on Control and Optimization, 40:1663-1696, 2002.

[9] E. J. Collins and J. M. McNamara. The job-search problem with competition: An evolutionary stable dynamic strategy. Adv. Appl. Prob., 25:314-333, 1993.

[10] S. Dayanik and I. Karatzas. On the optimal stopping problem for one-dimensional diffusions. Stochastic Processes and their Applications, 107 (2):173-212, 2003.

[11] E. Dynkin. Markov processes, Volume II. Springer Verlag, Berlin, 1965.

[12] I. Karatzas and S. E. Shreve. Browninan Motion and Stochastic Calculus, 2nd Edition. Springer-Verlag, New York, 1991.

[13] S. G. Kou and H. Wang. First passage times of a jump diffusion process. Adv. Appl. Prob., (35):504-531, 2003.

[14] S. A. Lippman and J. J. McCall. Job search in a dynamic economy. J. Econ. Thory, 12:365-390, 1976.

[15] E. Mordecki and P. Salminen. Optimal stopping of hunt and lévy processes. Stochastics, 79:233-251, 2007.

[16] T. Nakai. Properties of a job search problem on a partially observable markov chain in a dynamic economy. Computers and Mathematics with Applications, 51 (2):189-198, 2006. 
[17] B. Øksendal and A. Sulem. Applied Stochastic Control of Jump Diffusions. Springer, New York, 2005.

[18] S. M. Ross. Introduction to Stochastic Dynamic Programming. Academic Press, 1982.

[19] S. M. Ross. Introduction to Probability Models, 9th Edition. Academic Press, 2007.

[20] W. Stadje. A new continuous-time search model. J. Appl. Prob., 28:771-778, 1991.

[21] D. Zuckerman. Job search: The continuous case. J. Appl. Prob., 20:637-648, 1983.

[22] D. Zuckerman. On preserving the reservation wage property in a continuous job search. J. Econ. Theory, 34:175-179, 1984.

[23] D. Zuckerman. Optimal stopping in a continuous search model. J. Appl. Prob., 23:514-518, 1986. 